\title{
Incorporating animal movement into distance sampling
}

\section{R. Glennie, S. T. Buckland, R. Langrock, T. Gerrodette, L. T. Ballance, S. J.} Chivers \& M. D. Scott

To cite this article: R. Glennie, S. T. Buckland, R. Langrock, T. Gerrodette, L. T. Ballance, S. J. Chivers \& M. D. Scott (2020): Incorporating animal movement into distance sampling, Journal of the American Statistical Association, DOI: 10.1080/01621459.2020.1764362

To link to this article: https://doi.org/10.1080/01621459.2020.1764362

View supplementary material

Accepted author version posted online: 05 May 2020.

Submit your article to this journal

Llll Article views: 25

View related articles ־

View Crossmark data $\asymp$ 


\section{Incorporating animal movement into distance sampling}

R. Glennie ${ }^{\star 1 \#}$, S. T. Buckland ${ }^{1}$, R. Langrock ${ }^{2}$, T. Gerrodette ${ }^{3}$, L. T. Ballance ${ }^{3}$, S.

J. Chivers ${ }^{3}$, M. D. Scott ${ }^{4}$

1 University of St Andrews, Centre for Research into Ecological and

Environmental Modelling

2Bielefeld University

3Southwest Fisheries Science Centre, NOAA Fisheries

4Inter-American Tropical Tuna Commission, California

*Corresponding author: Richard Glennie. Email: rg374@st-andrews.ac.uk

\#R. Glennie gratefully acknowledges the Carnegie Trust for funding his work on

this research project

\section{Abstract}

Distance sampling is a popular statistical method to estimate the density of wild animal populations. Conventional distance sampling represents animals as fixed points in space that are detected with an unknown probability that depends on the distance between the observer and the 
animal. Animal movement can cause substantial bias in density estimation. Methods to correct for responsive animal movement exist, but none account for non-responsive movement independent of the observer. Here, an explicit animal movement model is incorporated into distance sampling, combining distance sampling survey data with animal telemetry data. Detection probability depends on the entire unobserved path the animal travels. The intractable integration over all possible animal paths is approximated by a hidden Markov model. A simulation study shows the method to be negligibly biased (less than $5 \%$ ) in scenarios where conventional distance sampling overestimates abundance by up to $100 \%$. The method is applied to line transect surveys (1999-2006) of spotted dolphins (Stenella attenuata) in the eastern tropical Pacific where abundance is shown to be positively biased by $21 \%$ on average, which can have substantial impact on the population dynamics estimated from these abundance estimates and on the choice of statistical methodology applied to future surveys.

Keywords: abundance; hidden Markov model; continuous-time; diffusion

\section{Introduction}

Distance sampling is a statistical method used to estimate the population density of wild animals (Buckland et al. 2015). It is applied to a wide variety of taxa, e.g., birds (Newson et al. 2008), cetaceans (Hammond et al. 2013), and mammals (Aars et al. 2009). Many conservation and management studies depend on the accuracy of distance sampling inference; yet, the statistical method relies on a key assumption that is substantially violated in some applications. 
Distance sampling is a snapshot method (Buckland et al. 2005): the survey is assumed to occur instantaneously. Animals are idealised as static points that are detected with unknown probability by an observer, who stands at a point or traverses a line within the study region. Surveyed transects, lines or points, are placed according to a randomised design such that animals are distributed independently of the observer. Furthermore, it is assumed animals are distributed independently of each other. For line transects, animals are distributed uniformly around the line, in point transects they follow a triangular distribution. Given this, the decline in the number of detections as distance from the observer increases is solely due to a change in the probability of detection; thus, the recorded locations are used to estimate this probability and, ultimately, animal density. In short, distance sampling is a thinned point process model with unknown thinning probability (Hedley and Buckland 2004, Yuan et al. 2017). The assumption that the survey is a temporal snapshot of the animal population is central to distance sampling theory; the method, however, is applied to surveys of mobile animal populations where transects are surveyed over a time interval within which animals may have moved a significant distance.

Animal movement can be in response to an observer's presence (Turnock and Quinn 1991), attraction or avoidance, or can be of the animal's own accord, independent of the observer. Responsive movement is a well-known problem, 
and specific survey techniques, searching further along line transects to see animals before they respond or remaining at point transects long enough for animals to resume normal behaviour, are recommended to mitigate bias in density estimates (Buckland et al. 2005). Furthermore, double-observer methods exist that can account for responsive movement (Conn et al. 2018, Borchers et al. 1998, Palka and Hammond 2001). In comparison, movement independent of the observer has received little attention. For point transects, a snapshot method is recommended to reduce bias in estimates (Buckland 2006); however, many surveys do not employ this method, and it does not suit technological advances where observation technology may survey a point for a considerable time. Splitting continuous surveying periods at points into discrete snapshots involves subjective judgments that can affect the inferences obtained (Howe et al. 2017). Alternatively, cue-counting, where animals' cues such as bird calls are counted rather than individuals, is used as it is negligibly biased by non-responsive animal movement, but this relies on the animal population having a clearly defined cue (Buckland et al. 2005). For line transects, a rule-of-thumb, based on a limited simulation study, deems surveys on animals that move at less than half the observer's speed to be free of substantial bias (Hiby 1982); yet, observer speed is often constrained by the transport chosen and the terrain covered. Thus, density can be unavoidably overestimated due to animal movement. This 
overestimation is not caused by counting the same animal more than once, but by more animals entering the transect from outside and recorded locations leading to a biased estimated detection function (Glennie et al. 2015). Surveys of mobile animals record greater numbers of unique individuals, compared to a hypothetically immobile population, and animals are recorded closer to the observer; both effects led to positive bias in density estimation (Glennie et al. 2015). This calls into question inference drawn from surveys where animal movement is undeniable, and precludes the use of distance sampling on populations of fast-moving animals and on studies where transects are surveyed over a long time period.

Previous work has considered only how movement affects the number of animals seen, not where they are seen (Yapp 1956). Random encounter models (Lucas et al. 2015), where animals are assumed to move in randomly-orientated straight lines at constant speed (Hutchinson and Waser 2007), can provide estimates of density, corrected for movement, given the count of animals seen and an independent estimate of animal speed. A detection probability can also be included, but must be ascertained independently. An advantage of distance sampling is that the detection probability can be estimated from the data. Yet, this probability, when movement is admitted, depends on the entire path the animal has travelled whilst the transect is surveyed. This path, other than the 
single location observed when the animal is detected, is unobserved. Thus, any estimation of detection probability that accounts for animal movement must include a continuous space-time hidden process to describe the animal's trajectory. Furthermore, to calculate the proportion of animals never seen, the method must average over all possible animal trajectories. This can be achieved by specifying an explicit model for the encounter process (Gurarie and Ovaskainen 2012).

Hidden Markov models (HMMs) (Zucchini et al. 2016) are used for time series data that arise from an unobserved (or partially observed) stochastic process. In particular, HMMs are used to analyse animal telemetry data (Pedersen et al. 2011), animal locations recorded over time, where the paths taken by animals between recorded locations are averaged over according to the movement model specified. Distance sampling observations consist of a single such recorded location where the animal's movement prior to detection is unobserved; thus, if independent information on animal movement is collected, distance sampling can be viewed as a HMM, where animal paths are a hidden process and detection is the observed process. Tagging and tracking of animals is becoming more common as the technology reduces in price and size. HMMs can allow this auxiliary information to improve distance sampling estimation. Pedersen et al. (2011) developed a spatial HMM with an unobserved, diffusive movement 
process which is described by a stochastic partial differential equation. The intractable likelihood in continuous space and time is approximated by discretising space into a large number of spatial cells (Eydeland 1994); the computations involved are costly, constraining the level of discretisation attainable.

Here, a spatial HMM that incorporates animal movement into distance sampling using independently obtained animal movement data is presented. The likelihood is formulated in continuous space-time and a discrete approximation is then described. A simulation study compares this method to conventional distance sampling, and the method is applied to a line transect survey of spotted dolphins (Stenella attenuata attenuata) in the eastern tropical Pacific (ETP) (Gerrodette and Forcada 2005, Gerrodette et al. 2008).

\section{Methods}

Suppose $n$ animals are detected in total over the survey. The goal is to estimate the total abundance in the survey region, $N$. In distance sampling surveys, observers search transects and record the location of any animal they encounter. In line transect sampling, the perpendicular distance from the line to each encounter is recorded; in point transects, the radial distance is recorded. Use of 
two-dimensional location data, forward distance on line transects and angle of detection on points, is seldom used (Borchers and Cox 2017). Furthermore, the time of a detection, though routinely recorded, is not used. Here, a model is developed that uses both pieces of information: for the $i^{\text {th }}$ detected animal, let $\vec{x}_{i}$ be the two-dimensional location of the animal when detected and $t_{i}$ be the time between the observer beginning to survey the transect that animal i was seen on and the time animal $i$ was detected.

Recording the single location an animal is encountered provides no information about how the animal moves: independent data is required on animal movement. Suppose $m$ animals are tracked or tagged and their movement paths recorded over time. It is assumed the movement of these tagged animals is representative of the movement of any animal in the study area. Note it is not required that the tagged animals be members of the surveyed population.

\subsection{CDS Model}

In conventional distance sampling (CDS), the probability density function (PDF) of the recorded animal locations is estimated. A detection function, $g(x)$, is defined as the conditional probability an animal is detected given it resides at distance $x$. For line transects, distance is defined as the perpendicular distance 
the animal is from the line; for point transects, it is defined as the radial distance from the point. The probability density of the observed distances is then given by

$$
f(x)=\frac{g(x) \lambda(x)}{\int_{p} g(x) \lambda(x) \mathrm{d} x}
$$

where $^{\mathcal{P}}$ is the set of all possible animal locations and $\lambda$ is the probability density function of the animal's location. CDS makes the design-based assumption that transects are placed according to a randomised scheme. This implies for line transects that $\lambda$ is the density of a uniform distribution over ${ }^{\mathcal{P}}$ and for point transects a triangular distribution over ${ }^{\mathcal{P}}$. In short, $\Lambda$ is known and $g$ is to be estimated.

A convenient functional form is chosen for the detection function and its parameters estimated by maximum likelihood. This form is chosen ad hoc to be half-normal or exponential with some trigonometric or polynomial adjustments. Alternatively, an explicit model for the detection process can be specified by a two-dimensional hazard-rate function ${ }^{h(x, t)}$ (Borchers and Cox 2017, Skaug and Schweder 1999), which describes the detection rate of an animal residing in location ${ }^{x}$ at time $t$. This is equivalent to a survival process where death is interpreted as detection and the detection intensity varies over time and space. In practice, CDS analyses do not use the time of detections. Instead, a detection function is derived from a given hazard by integrating over the time interval that 
an animal is at risk of detection. Nonetheless, here, the term CDS is used to refer to $2 \mathrm{D}$ hazard models also. The hazard commonly depends on the radial distance between the observer and the animal, $r(\vec{x}, t)$, such that the hazard is infinite at zero radius and decreases with increasing radius.

\subsection{MDS Model}

In this paper, the movement with distance sampling (MDS) model is introduced and presented as an extension of the existing CDS approach, thereby incorporating animal movement modelling with distance sampling.

Here, the recorded detection times and the two-dimensional recorded location are used to estimate the search process. Detection times are required since animals recorded at later times on the transect have had longer to move and so may have originated at a further distance. Rather than condition on the animal residing at a single fixed point, the detection probability is derived conditional on the animal travelling a fixed path over space. Given an animal travels a path $\mathbf{x}$ and is seen at location $\vec{x}_{\tau}$ at time $\tau$, the conditional PDF is $g(\mathbf{x}, \tau)=S_{\tau}(\mathbf{x}) h\left(\vec{x}_{\tau}, \tau\right)$ where $S_{t}(\overrightarrow{\mathbf{x}})=\exp \left\{-\int_{0}^{t} h\left(\vec{x}_{s}, s\right) \mathrm{d} s\right\}$ is the probability of the animal eluding detection 
until time $t$. Notice, detection probability now depends on time and the entire trajectory of the animal.

Similar to CDS, if in place of assuming the distribution of animals, we assume the movement process is known, and in place of conditioning on the location of an animal, we condition on the path an animal has taken, the PDF of the observed encounter on a transect of duration $T$ is given by:

$$
f(\overrightarrow{\mathbf{x}}, \tau)=\frac{\overrightarrow{g(\mathbf{x}, \tau) \lambda(\overrightarrow{\mathbf{x}})}}{\int_{\chi} \int_{0}^{T} g(\overrightarrow{\mathbf{y}}, t) \mathrm{d} t \mathrm{~d} \Lambda(\mathbf{y})}
$$

where $\Lambda$, with associated density $\lambda$, is the probability measure over all (measurable) paths, $X$, that an animal could have taken. The denominator is the probability an animal is seen at some time on the transect and is required as we do not observe those animals that were never encountered.

Yet, the paths of animals are unobserved and conventional distance sampling surveys provide no information on how animals move. Thus, independent animal movement data is required to determine $\Lambda$. Here, animal movement is described by Brownian motion with rate parameter $v$. This makes $\Lambda$ mathematically tractable: any set of observed locations on a movement path have a multivariate Gaussian distribution. For telemetry data, one can condition on the initial location of the animal and compute the likelihood, ${ }^{\mathcal{L}}$, easily (Okubo and Levin 2013); for 
distance sampling surveys, the initial locations are assumed to be independently distributed with respect to the transect.

From the distance sampling survey, given animal $i$ was recorded in location $\vec{x}_{i}$ at time $\tau$, the likelihood for the detection parameters, ${ }^{\theta}$, is obtained by averaging over all possible animal paths:

$\mathcal{L}_{\theta}=\prod_{i=1}^{n} \frac{\int_{\chi_{i}} g\left(\overrightarrow{\mathbf{x}}, \tau_{i}\right) \mathrm{d} \Lambda(\overrightarrow{\mathbf{x}})}{p_{i}}$

where $X_{i}$ is the space of all measurable paths that pass through location $\vec{x}_{i}$ at time $\tau_{i}$, and ${ }^{p_{i}=1-\int_{\chi} S_{T_{i}}(\mathbf{x}) \mathrm{d} \Lambda(\mathbf{x})}$ is the probability the encounter with animal $i$ occurs at some time when the transect is surveyed for total time $T_{i}$.

Assuming the distance sampling survey and the animal telemetry data are independent, the combined likelihood $\mathcal{L}_{\theta, 2}=\mathcal{L}_{\theta} \mathcal{L}_{2}$ can be maximised to obtain maximum likelihood estimates, ${ }^{(\theta, \lambda)}$. Methods in section 2.3 describe the approximations used to compute the likelihood. Maximising the combined likelihood means that uncertainty in the movement parameters is propagated to the density estimation. Abundance can be estimated using either approach already available in distance sampling: a model-based estimator or a HorvitzThompson-like estimator (Buckland et al. 2016). 
The Horvitz-Thompson-like estimator of abundance is

$$
\hat{N}=\frac{n}{\sum_{k=1}^{K} \hat{p}_{k}}
$$

where $K$ is the total number of transects, $n$ is the number of animals seen, and $\hat{p}_{k}$ is the estimated probability of detection on transect $k$. A sandwich estimator for the variance of $\hat{N}$ can be derived analogously to the estimator used in CDS (Fewster et al. 2008).

Alternatively, an explicit model for abundance can be integrated (Buckland et al. 2016), for example, the Poisson process: $\mathcal{L}_{N}=\left(N p_{\text {enc }}\right)^{n} \exp \left(-N p_{\text {enc }}\right) / n !$ where $p_{\text {enc }}$ is the probability of detection during the survey. This gives a model-based estimate of abundance by maximising the likelihood ${ }^{\mathcal{L}}{ }_{\theta, \lambda, N}=\mathcal{L}_{\theta} \mathcal{L}_{\lambda} \mathcal{L}_{N}$.

\subsection{Computation}

The likelihood, formulated in continuous space and time, is analytically intractable. In this section, existing approximations to a similar type of model are adapted to this context to make the computations practical. The integral over all possible animal paths can be approximated by quadrature. A buffer region around each transect is discretised into $K$ cells of length and width ${ }^{\Delta x}$ and time 
is discretised into steps of duration ${ }^{\Delta t}$. Animal movement occurs at these timesteps, thus animals are stationary within time-steps.

Notice that all integrals to be approximated are of the form $\int_{x} S_{t}(\vec{x}) \mathrm{d} \Lambda(\vec{x})$ for some time $t$. Approximating animal movement by jumps at each time-step, the function $S$ can be separated into components:

$S_{t}(\overrightarrow{\mathbf{x}})=s_{0}\left(\vec{x}_{0}\right) s_{\Delta t}\left(\vec{x}_{\Delta t}\right) s_{2 \Delta t}\left(\vec{x}_{2 \Delta t}\right) \ldots s_{t-\Delta t}\left(\vec{x}_{t-\Delta t}\right)$

where ${ }^{s_{l}(\vec{x})=\exp \left(-\int_{l}^{l+\Delta t} h(\vec{x}, u) \mathrm{d} u\right)}$ is the probability of eluding detection during a single time-step given the animal is at location ${ }^{x}$ Assuming animals are stationary within time-steps, ${ }^{s_{l}(x)}$ can be computed analytically within each timestep, accounting for the continuous movement of the observer, thus only animal movement is discretised. Let $\mathbf{P}$ be a $K \times K$ diagonal matrix with $k^{\text {th }}$ diagonal $s_{t}\left(\vec{y}_{k}\right)$ where $\vec{y}_{k}$ is the centre location of spatial cell $k$.

The integration over all paths is replaced with a sum over all paths on the discrete grid. When discretised, Brownian motion can be approximated by a continuous-time Markov chain (Pedersen et al. 2011). Pedersen et al. (2011) derive the transition rate matrix, $\mathbf{G}$, using a finite difference approximation to the Fokker-Planck equation (Okubo and Levin 2013, Mitchell and Griffiths 1980). By 
taking the matrix exponential, the transition probability matrix for a given time step $\Delta t$ can be computed, $\Gamma=\exp (\mathbf{G} \Delta t)$ (Moler and Van Loan 2003).

The integral is approximated by a spatial HMM likelihood (Zucchini et al. 2016, Pedersen et al. 2011):

$\int_{x} S_{t}(\mathbf{X}) \mathrm{d} \Lambda=\mathbf{p}_{0} \mathbf{P}_{0} \Gamma \mathbf{P}_{\Delta t} \Gamma \ldots \mathbf{P}_{t} \Gamma \mathbf{1}$

where $\mathbf{p}_{0}$ is $1 \times K$ row vector representing the initial distribution of animals on the grid with respect to the transect and 1 is a $K \times 1$ column vector of ones.

In distance sampling surveys, the range of detection distances can be small compared to the distances an animal can travel during a survey period. The former makes it necessary that $\Delta x$ be adequately small while the latter requires the buffer around the transect, and so the grid, to be large. Ultimately, $K$ is large. This makes the matrix calculations in equation (2) computationally demanding. The sparse structure of $\mathbf{G}$ can be exploited to accelerate computations using the Krylov subspace approximation (Hochbruck and Lubich 1997) and, when movement is isotropic and G block-Toeplitz, the 2D discrete Fourier transform (Lee 1986). For spatial HMMs, there is no analytical bound on the error of this approximation. Practical advice is to reduce the discretisation until the path integral's value no longer significantly changes, e.g., in relative percentage. 


\subsection{Simulation study}

A simulation study is conducted to demonstrate the performance of MDS compared to CDS for two particular distance sampling surveys. The magnitude of the bias in CDS depends on the relationship between relative animal speed, transect width, and the shape of the detection function (Glennie et al. 2015). This simulation study considers the effect of animal speed when all other factors are fixed. The simulation was conducted using the moveds 0.1 .0 package within the $\mathrm{R}$ programming environment ( $\mathrm{R}$ Core Team 2017). The code to perform these simulations is included in the supplementary materials.

A study population of 100 animals in 100 square kilometres is simulated. Animals move according to Brownian motion with average rate changing for each simulation scenario. The independent animal telemetry data required was simulated from Brownian motion, recording the location of ten tagged animals every minute for one hour.

Two distance sampling surveys were simulated on this population: a line transect study and a point transect study. The line transect survey consists of 50 transects of length 1 kilometre; the observer traverses each line at speed 1 metre per second. For these simulation scenarios, animals moved at speeds covering $50 \%$ to $300 \%$ the speed of the observer. For the point transect survey, 100 points 
were surveyed, each for 5 minutes, with animals speeds from 0.5 metres per second to 4.0 metres per second.

In both surveys, the hazard of detecting an animal at a radial distance $r$ is given

by $\left(\frac{r}{s}\right)^{-(d+1)}$ for scale parameter $s>0$ and shape parameter $d>0$ (Hayes and Buckland 1983, Borchers and Cox 2017). Detection parameters were chosen as $s=5, d=2$, such that for a hypothetically immobile animal population, the effective area searched was approximately 0.015 square kilometres. This corresponds to a line transect with half-width 30 metres and point transect with radius 100 metres.

One hundred simulations were performed. A distance sampling model with no animal movement was fit to each simulated data set. For fair comparison, a twodimensional hazard was used in this model also, unlike CDS models where a one-dimensional hazard is commonly used. An MDS model, as presented in this paper, was fit to each data set with the auxiliary movement data using a grid size of 2.5 metres and a time-step of 1 second. The relative bias, mean square error and confidence interval coverage was estimated for each scenario, with and without movement incorporated.

\subsection{Application: spotted dolphins}


The presented method is applied to shipboard line transect surveys conducted in the eastern tropical Pacific on spotted dolphins in 1999, 2000, 2003, and 2006. Here, the abundance within the core area, as defined by Gerrodette and Forcada (2005), is estimated.

Dolphin schools are treated as the individual unit of detection and group size estimated separately. This is a standard approach in CDS; however, incorporating movement makes the approach more questionable. In particular, we assume that schools do not fuse or break-up during the time the observer surveys each transect. Furthermore, we assume the movement model, informed by tags on single individuals, describes the movement of a school as a whole. Independent tag data was collected on nineteen spotted dolphins (Scott and Chivers 2009) providing fixed locations at approximately 15 minute intervals over 1-2 days.

The radial distance and angle to each detected spotted dolphin school was recorded. Only sightings in Beaufort state 3 or less were retained, as in the higher Beaufort states detectability declines more rapidly with distance. The location of the ship was recorded every ten minutes. It is assumed the ship travels in a straight line at a constant speed between these records. The movement model does not account for movement caused by ocean current; it is 
assumed that the animals and ship are drifting in the same direction and at the same rate, thus this movement has no effect on their relative positions.

Three methods were applied to the data: 1D conventional distance sampling (CDS1D), 2D conventional distance sampling (CDS2D), and 2D distance sampling with movement (MDS2D). CDS1D is the standard modelling framework used for distance sampling analysis; it was applied to these data using the $R$ package Distance 0.9.7 (Miller et al. 2019). The two-dimensional models were fit using the $\mathrm{R}$ package moveds 0.1 .0 with a grid size of 0.2 kilometres and time-step of 10 minutes. Only the hazard-rate model with no trigonometric or polynomial adjustments was considered for the CDS1D model as then the same hazard is assumed for all three methods: $\left(\frac{r}{s}\right)^{-(d+1)}$ with parameters $s, d>0$. A truncation distance of 5.5 kilometres was used, as used in Gerrodette and Forcada (2005), as this is the maximum distance a sighting could occur for confirmed species identification. Dolphin school abundance is estimated using the Horvitz-Thompson-like estimator given above. Goodness of fit is evaluated using a Cramér-Von-Mises test (Cramér 1928), comparing the expected distribution of perpendicular distances to the observed (Buckland et al. 2005).

\section{Results}




\subsection{Simulation study}

\subsubsection{Line transect simulation}

The CDS estimator of abundance had bias $>10 \%$ when animal speed exceeded 1.25 metres per second and CDS overestimated abundance by $>100 \%$ for speed $>3.0$ metres per second. In contrast, MDS led to $<5 \%$ bias for all scenarios (Figure 1). Mean square error (MSE) for CDS estimators was dominated by their bias; MDS showed constant MSE across all animal speeds. Confidence interval coverage across all parameters for CDS was less than $40 \%$ for speeds over 1.0 metre per second and fell to $0 \%$ for speeds over 2.0 metres per second. MDS coverage was nominal within $1 \%$ for all parameters and across all simulation scenarios.

\subsubsection{Point transect simulation}

CDS point transect sampling behaved similarly with bias $>10 \%$ for animal speed $>2$ metres per second and bias reaching $90 \%$ for speed around 4 metres per second. Incorporating movement reduced bias to $<5 \%$ across all scenarios and mean square error varied negligibly. CDS $95 \%$ confidence interval coverage was poor $(<45 \%)$ for all parameters when animal speed exceeded 2 metres per second, while coverage was nominal for all parameters when movement was incorporated. 


\subsection{Application: spotted dolphins}

Estimated abundance of spotted dolphin schools in the core area, as defined by Gerrodette and Forcada (2005), differed substantially between models with and without movement (Table 1). One and two dimensional CDS models led to similar estimates of abundance. Incorporating movement reduced the abundance estimate by approximately $21 \%$ on average across the years. The coefficient of variation $(\mathrm{CV})$ for the abundance estimator was similar between the twodimensional models. The average speed of the ship was $17 \mathrm{~km} / \mathrm{h}$; the estimated average speed of each spotted dolphin was $7.4 \mathrm{~km} / \mathrm{h}$. The large reduction in the abundance estimate indicates that even though the dolphins move relatively slowly compared to the ship, bias can be substantial, because, whilst being surveyed, they can move a large distance compared to the width of the transect. This highlights the danger of assessing whether movement is a problem based solely on relative animal speed; MDS can account for the interdependent effects of animal speed, transect width, and detection function shape.

For comparison, the expected number of sightings within each 0.5 kilometre from the transect line was calculated (Figure 3). CDS1D, CDS2D, and MDS2D models all had similar goodness-of-fit (Cramer-von-mises p-value > 0.05); however, the 
estimated detection function differs considerably when animal movement is included (Figure 4). The CDS1D and CDS2D estimated detection functions decline more rapidly with distance than the MDS2D function indicating that animal movement has caused negative bias in the estimation of detection probability. This effect, together with the greater number of animals that move into the transect and are seen, causes abundance to be overestimated. Note, these deficiencies do not result in CDS models failing a test of goodness-of-fit to the observed data, but has an important effect on the final abundance estimate.

\section{Discussion}

Distance sampling surveys on mobile animal populations should not ignore animal movement. The simulation study demonstrates the remarkable bias that non-responsive animal movement can cause. Incorporating this movement into distance sampling can mitigate this bias and remove the subjective judgment of when movement bias may have occurred and to what extent.

The application to spotted dolphin surveys of the Eastern Tropical Pacific (ETP) further shows that abundance estimates can be biased by around $20 \%$ when movement independent of the observer is not accounted for. Since 2006, there have been no further National Marine Fisheries Service surveys, and so the 
abundance estimates from the surveys considered here (1999-2006) provide the most recent information on the population dynamics of this dolphin population; these abundance estimates are used to estimate mortality rate and by-catch from the tuna fisheries industry (Gerrodette and Forcada 2005). Lennert-Cody et al. (2019), in response to the growing need to gather more up-to-date information on these populations, present a review of current methodology and survey methods possible for future ETP surveys where they identify ship-based surveys as the most viable immediate option; significantly, the review also highlights the presence of bias due to independent animal movement and the need to apply methods that can account for it.

The cost of this improvement in estimation is the need for additional information on animal movement. The expense and practicality of collecting such data depends on the species to be surveyed. For example, tag data on cetaceans, ungulates, and seabirds is becoming more common. When no such data is available, a 'plug-in' estimator of the movement parameters could be used, similar to the multipliers used in CDS. Accounting for movement on expert, though imperfect, knowledge may be better than ignoring it completely. The movement information need not come from animals in the same population as that surveyed by distance sampling, nor be collected in the same time period. Nevertheless, it is assumed tagged animals behave as representative members 
of the study population; thus, it is recommended that movement information be collected around the same time the distance sampling survey is conducted and on animals that are members of the study population.

Estimating a detection process that depends on time necessarily requires detection times be recorded. In practice, this information is often recorded in the field, but not used in CDS models. Here, this information is essential and any application of the model would require this data to be collected. Records of the observer's location over time must also be kept. Furthermore, the location of detected animals should be recorded relative to the observer; this is contrary to practice in CDS where measurements are made relative to the transect. Also, animal location must be recorded in two-dimensional space.

The main obstacle to this method is the computational burden. The case study shows that the approximations presented allow for real survey data to be analysed in a practical time; however, the computational demand may limit application when surveyed transects are large compared to the scale of detection or when survey times are long. An alternative to the quadrature-based approach presented here is to simulate: animal paths are simulated and the average likelihood taken as a Monte Carlo estimate of the marginal likelihood, averaged over all paths. The appendix to this paper compares simulation to the 
quadrature-style approach and highlights the problems to be tackled in future work.

\section{Assumptions}

The assumptions made in the theory presented are synonymous with those made in CDS (Buckland et al. 2015). Violations of these assumptions will cause bias in the inference obtained. Good survey design and protocol can be used to reduce the extent to which assumptions are violated, see Buckland et al. (2015). As with the assumption of no animal movement in CDS, there exist analytical methods that remove other necessary assumptions of distance sampling, often with the requirement that additional data be collected. MDS, as an extension of the CDS framework, can accommodate these theoretical extensions when the assumptions below are not adequately met.

1. The path an animal travels is independent of the observer: animals do not respond to the observer and their movement is independent of the transect placement, that is, surveying does not preferentially take place in areas animals would avoid or be attracted to.

2. Animals at zero radius are detected: this assumption can be violated for animals that are not always available for detection, for example, a diving 
cetacean can be missed by an observer in a ship directly above it (Langrock et al. 2013, Borchers et al. 2013).

3. Location measurements are exact: this assumption applies to observed locations of animals on the distance sampling survey and the recorded locations of tracked animals. Observation error in animal telemetry data is common and can be accounted for (Johnson et al. 2008). Models for measurement error in distance sampling can also be incorporated (Marques 2004).

4. Animal movement is diffusive: the simple model that animal movement is a spatially-invariant, isotropic diffusion process is violated by many animal populations; more realistic movement models can be considered. No matter what movement model is incorporated, one assumes that all animals in the survey move according to the specified model. Departures from the movement model could cause detection probability to be biased. In particular, underestimating or overestimating animal speed will lead to overestimating or underestimating density, respectively. Glennie et al. (2015) also shows that if animal movement is less tortuous than assumed under Brownian motion, then assuming a Brownian motion movement model will lead to overestimation in abundance; however, this overestimation will be reduced compared to CDS. 
5. Sampling is representative and independent: for the distance sampling survey, this assumption requires transects be placed according to a randomised design, that transects be independent, and that animals be independent. For animals that travel in groups, treating groups as the independent unit to be sampled may be a better choice. For the movement model, it is assumed that tagged animals move independently and that the sample of tagged animals be representative of the surveyed population. One can use telemetry from tagged animals who are not members of the surveyed population, but only with the assumption these animals exhibit movement patterns similar to those animals surveyed by distance sampling. Despite this, due to the heterogeneity of animal behaviour, it is best to collect auxiliary movement data on the same population being surveyed and at the same time the survey takes place.

\section{Model extensions}

The model formulation is flexible and can include existing extensions of conventional distance sampling. Here, only a hazard that depends on radial distance was considered. A hazard that depends on angle and radius could be used. For point transects, any detection function can be used to define a hazard 
(Borchers et al. 2015). Yet, for line transects, the method relies on an analytical form for $S_{t}$ observer movement makes integration of the hazard more complicated. This could be avoided by discretising observer movement similarly to animal movement, that is, the observer only moves at time-steps and not within time-steps; however, this can introduce significant bias when discretisation is rough and often requires much lower levels of discretisation than otherwise necessary.

Another popular extension is multiple-covariate distance sampling (Marques and Buckland 2003). Including covariates in the detection process could be handled similarly with this model where parameters in the hazard can depend on environmental conditions over space and time. Given this, it is important to highlight that any covariates included would need to be known for each time and each location in space. This may require one to assume that covariates are constant in the time between their recording. Additional information on the movement of detected individuals can also be incorporated. Observed locations or observed directions of travel can be used to improve estimation of the unknown path each animal took. Furthermore, responsive movement could be accounted for if the response, how an animal's location changes over time with respect to the observer, is recorded for one or more focal individuals. 
Including covariate information in the movement model is more computationally demanding. The HMM computational algorithm used depends on the blockToeplitz structure of the transition rate matrix, G. A block-Toeplitz structure is equivalent to assuming that the transition rates are spatially invariant. Relaxing this assumption increases computation time and limits the level of discretisation that can be practically obtained.

Additionally, this assumption limits the range of possible movement models that can be considered. Bias in density estimation from animal movement is at its worst when animal movement is persistent in a single direction. A movement process with persistent movement would require the discretisation of a 4dimensional space: location and velocity. Given current computational resources, it is likely any practical discretisation of this space would be too coarse to provide good estimators of detection parameters. Nevertheless, for many taxa, animal movement is more complex than can be described by a diffusion process, and so extending MDS to include advection-diffusion or Ornstein-Uhlenbeck animal movement models could improve the inference obtained.

Finally, the methods presented can be extended to double-observer distance sampling, where two observers survey the transect simultaneously. If multiple sightings of an individual by different observers can be matched together, then only animal paths that pass through these multiple observed locations need be 
considered, providing information on animal movement directly from the distance sampling data and improving the estimation of each animal's detection probability.

\section{Conclusion}

Animal movement can be incorporated with distance sampling. The presented theoretical framework provides a basis for further development and the computational approach discussed makes the method applicable. Accounting for animal movement can mitigate the bias it causes in the surveys where distance sampling is applied, and it can widen the application of distance sampling to animals whose movement has so far prohibited its use.

\section{SUPPLEMENTARY MATERIAL}

S1 Comparison of simulation-based and quadrature-based computation of the path integral. (pdf)

S2 R code to conduct point transect and line transect example MDS analyses and simulation studies using moveds $\mathrm{R}$ package. (zip)

moveds 0.1.0 R package that implements the movement with distance sampling methods (GNU zipped tar file) 


\section{References}

Aars, J., Marques, T., Buckland, S., Andersen, M., Belikov, S., Boltunov, A. and Wiig, $\varnothing$. (2009), 'Estimating the Barents Sea polar bear subpopulation size', Marine Mammal Science 25(1), 35-52.

Borchers, D. L. and Cox, M. J. (2017), 'Distance sampling detection functions: 2d or not 2d?', Biometrics 73(2), 593-602.

Borchers, D. L., Stevenson, B. C., Kidney, D., Thomas, L. and Marques, T. A. (2015), 'A unifying model for capture-recapture and distance sampling surveys of wildlife populations', Journal of the American Statistical Association 110(509), 195-204.

Borchers, D. L., Zucchini, W. and Fewster, R. M. (1998), 'Mark-recapture models for line transect surveys', Biometrics pp. 1207-1220.

Borchers, D. L., Zucchini, W., Heide-Jørgensen, M. P., Cañadas, A. and Langrock, R. (2013), 'Using hidden Markov models to deal with availability bias on line transect surveys', Biometrics 69(3), 703-713.

Buckland, S. T. (2006), 'Point-transect surveys for songbirds: robust methodologies', The Auk 123(2), 345-357. 
Buckland, S. T., Anderson, D. R., Burnham, K. P. and Laake, J. L. (2005), Distance Sampling, Wiley Online Library.

Buckland, S. T., Oedekoven, C. S. and Borchers, D. L. (2016), 'Model-based distance sampling', Journal of Agricultural, Biological, and Environmental Statistics 21(1), 58-75.

Buckland, S. T., Rexstad, E. A., Marques, T. A. and Oedekoven, C. S. (2015), Distance Sampling: Methods and Applications, Springer.

Conn, P. B., Alisauskas, R. T. et al. (2018), 'Simultaneous modelling of movement, measurement error, and observer dependence in mark-recapture distance sampling: An application to arctic bird surveys', The Annals of Applied Statistics 12(1), 96-122.

Cramér, H. (1928), 'On the composition of elementary errors: First paper: Mathematical deductions', Scandinavian Actuarial Journal 1928(1), 13-74.

Eydeland, A. (1994), 'A fast algorithm for computing integrals in function spaces: financial applications', Computational Economics 7(4), 277-285.

Fewster, R. M., Buckland, S. T., Burnham, K. P., Borchers, D. L., Jupp, P. E., Laake, J. L. and Thomas, L. (2008), 'Estimating the encounter rate variance in distance sampling', Biometrics 65(1), 225-236. 
Gerrodette, T. and Forcada, J. (2005), 'Non-recovery of two spotted and spinner dolphin populations in the eastern tropical pacific ocean', Marine Ecology Progress Series 291, 1-21.

Gerrodette, T., Watters, G., Perryman, W. and Ballance, L. (2008), Estimates of 2006 dolphin abundance in the eastern tropical pacific, with revised estimates from 1986-2003, Technical report, NOAA, NMFS Technical Memorandum NOAA-TM-NMFS-SWFSC-422.

Glennie, R., Buckland, S. T. and Thomas, L. (2015), 'The effect of animal movement on line transect estimates of abundance', PloS one 10(3), e0121333.

Gurarie, E. and Ovaskainen, O. (2012), 'Towards a general formalization of encounter rates in ecology', Theoretical Ecology 6(2), 189-202.

Hammond, P. S., Macleod, K., Berggren, P., Borchers, D. L., Burt, L., Cañadas, A., Desportes, G., Donovan, G. P., Gilles, A., Gillespie, D. et al. (2013), ' Cetacean abundance and distribution in european atlantic shelf waters to inform conservation and management', Biological Conservation 164, 107-122.

Hayes, R. J. and Buckland, S. T. (1983), 'Radial-distance models for the linetransect method', Biometrics pp. 29-42. 
Hedley, S. L. and Buckland, S. T. (2004), 'Spatial models for line transect sampling', Journal of Agricultural, Biological, and Environmental Statistics 9(2), 181-199.

Hiby, A. (1982), 'The effect of random whale movement on density estimates obtained from whale sighting surveys', Rep. Int. Whal. Commn. 32, 791-794.

Hochbruck, M. and Lubich, C. (1997), 'On Krylov subspace approximations to the matrix exponential operator', SIAM Journal on Numerical Analysis 34(5), 19111925.

Howe, E. J., Buckland, S. T., Després-Einspenner, M. L. and Kuhl, H. S. (2017), ' Distance sampling with camera traps', Methods in Ecology and Evolution 8(11), $1558-1565$.

Hutchinson, J. M. C. and Waser, P. M. (2007), 'Use, misuse and extensions of “ ideal gas" models of animal encounter', Biological Reviews 82(3), 335-359.

Johnson, D. S., London, J. M., Lea, M.-A. and Durban, J. W. (2008), ‘ Continuous-time correlated random walk model for animal telemetry data', Ecology 89(5), 1208-1215.

Langrock, R., Borchers, D. L. and Skaug, H. J. (2013), 'Markov-modulated nonhomogeneous poisson processes for modeling detections in surveys of 
marine mammal abundance', Journal of the American Statistical Association 108(503), 840-851.

Lee, D. (1986), 'Fast multiplication of a recursive block Toeplitz matrix by a vector and its application', Journal of Complexity 2(4), 295-305.

Lennert-Cody, C. E., Buckland, S. T., Gerrodette, T., Webb, A., Barlow, J., Fretwell, P. T., Maunder, M. N., Kitakado, T., Moore, J. E., Scott, M. D. et al. (2019), 'Review of potential line-transect methodologies for estimating abundance of dolphin stocks in the eastern tropical pacific', Journal of Cetacean Research and Management.

Lucas, T. C., Moorcroft, E. A., Freeman, R., Rowcliffe, J. M. and Jones, K. E. (2015), 'A generalised random encounter model for estimating animal density with remote sensor data', Methods in Ecology and Evolution 6(5), 500-509.

Marques, F. F. C. and Buckland, S. T. (2003), 'Incorporating covariates into standard line transect analyses', Biometrics 59(4), 924-935.

Marques, T. A. (2004), 'Predicting and correcting bias caused by measurement error in line transect sampling using multiplicative error models', Biometrics 60(3), 757-763. 
Miller, D., Rexstad, E., Thomas, L., Marshall, L. and Laake, J. (2019), 'Distance sampling in R', Journal of Statistical Software, Articles 89(1), 1-28. URL: https://www.jstatsoft.org/v089/i01

Mitchell, A. R. and Griffiths, D. F. (1980), The finite difference method in partial differential equations, John Wiley.

Moler, C. and Van Loan, C. (2003), 'Nineteen dubious ways to compute the exponential of a matrix, twenty-five years later', SIAM review 45(1), 3-49.

Newson, S. E., Evans, K. L., Noble, D. G., Greenwood, J. J. and Gaston, K. J. (2008), 'Use of distance sampling to improve estimates of national population sizes for common and widespread breeding birds in the uk', Journal of Applied Ecology 45(5), 1330-1338.

Okubo, A. and Levin, S. A. (2013), Diffusion and Ecological Problems: Modern Perspectives, Vol. 14, Springer Science \& Business Media.

Palka, D. and Hammond, P. (2001), 'Accounting for responsive movement in line transect estimates of abundance', Canadian Journal of Fisheries and Aquatic Sciences 58(4), 777-787. 
Pedersen, M. W., Patterson, T. A., Thygesen, U. H. and Madsen, H. (2011), ‘ Estimating animal behavior and residency from movement data', Oikos 120(9), $1281-1290$.

R Core Team (2017), R: A Language and Environment for Statistical Computing, R Foundation for Statistical Computing, Vienna, Austria. URL: https://www.Rproject.org/

Scott, M. D. and Chivers, S. J. (2009), 'Movements and diving behavior of pelagic spotted dolphins', Marine Mammal Science 25(1), 137-160.

Skaug, H. J. and Schweder, T. (1999), 'Hazard models for line transect surveys with independent observers', Biometrics 55(1), 29-36.

Turnock, B. J. and Quinn, T. J. (1991), 'The effect of responsive movement on abundance estimation using line transect sampling', Biometrics 47(2), 701-715.

Yapp, W. B. (1956), 'The theory of line transects', Bird Study 3(2), 93-104.

Yuan, Y., Bachl, F. E., Lindgren, F., Borchers, D. L., Illian, J. B., Buckland, S. T., Rue, H., Gerrodette, T. et al. (2017), 'Point process models for spatio-temporal distance sampling data from a large-scale survey of blue whales', The Annals of Applied Statistics 11(4), 2270-2297. 
Zucchini, W., MacDonald, I. L. and Langrock, R. (2016), Hidden Markov models for time series: an introduction using R, Chapman and Hall/CRC. 
Fig. 1 Percentage relative bias in estimated density for conventional distance sampling (solid line) and distance sampling with movement incorporated (dotted line) against animal speed (as \% of observer speed) estimated from 100 simulations of a line transect survey of 50 transects with truncation width 30 metres and observer speed 1 metre per second. Animals move according to Brownian motion. Shaded region marks $\pm 5 \%$ relative bias.

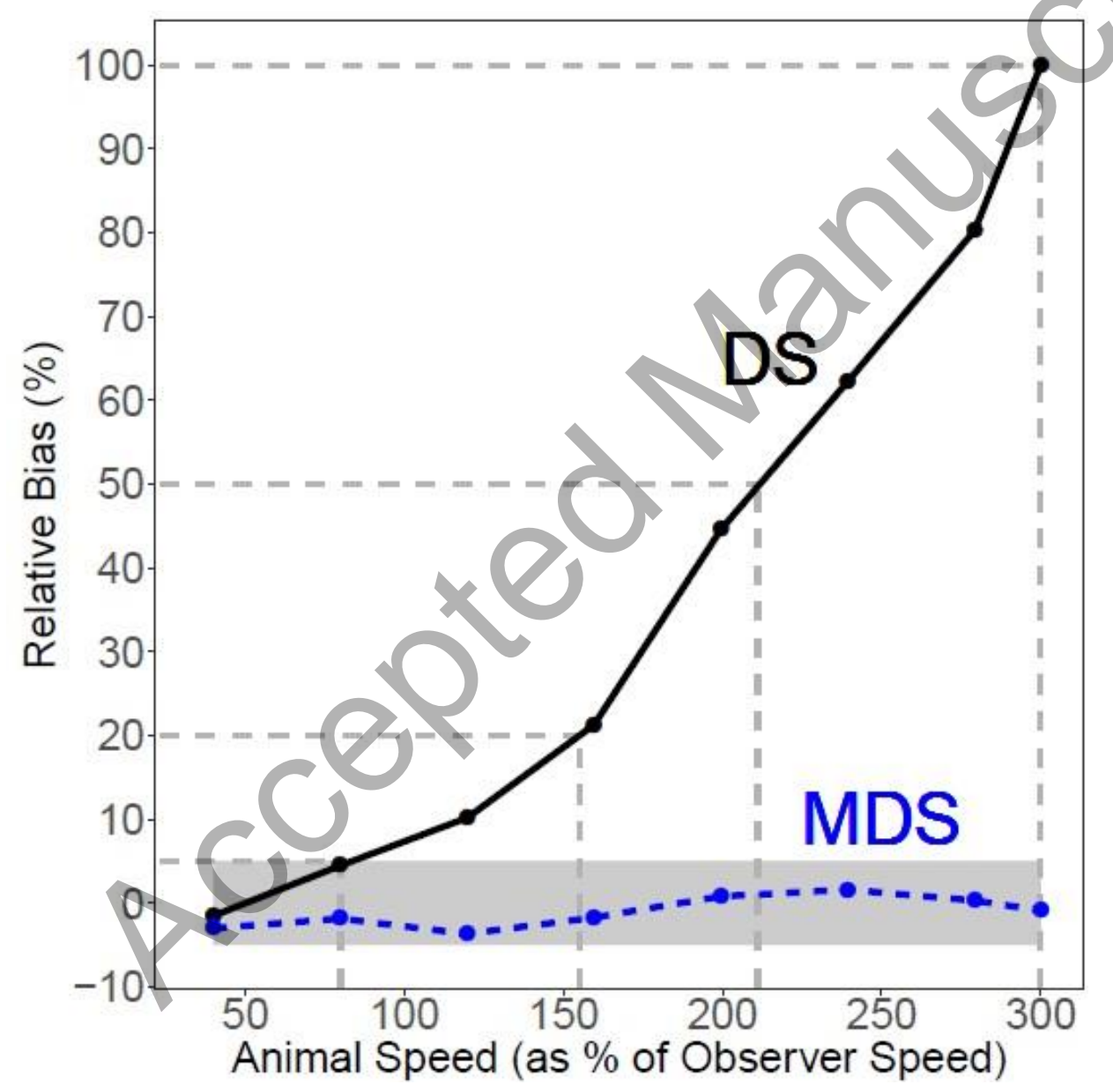


Fig. 2 Percentage relative bias in estimated density for conventional distance sampling (solid line) and distance sampling with movement incorporated (dotted line) against animal speed estimated from 100 simulations of a point transect survey of 100 transects with truncation width 100 metres, surveyed for 5 minutes. Animals move according to Brownian motion. Shaded region marks $\pm 5 \%$ relative bias.

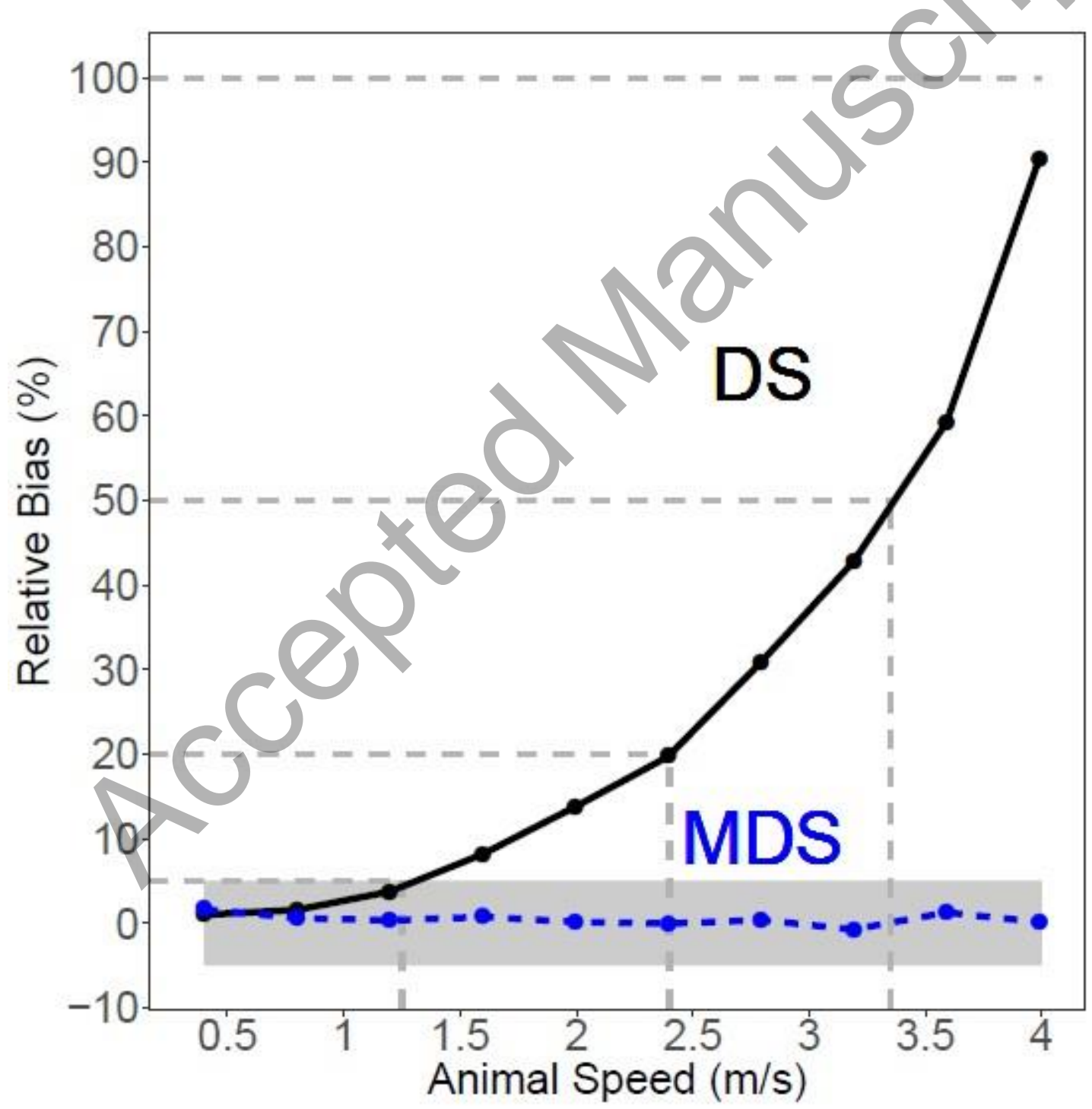


Fig. 3 Observed number of spotted dolphin schools sighted in each $0.5 \mathrm{~km}$ perpendicular distance from the transect line (shaded bars) with expected number of sightings from conventional distance sampling model for 1D (solid lines) and 2D (dashed lines) models and distance sampling with movement model (dotted lines).

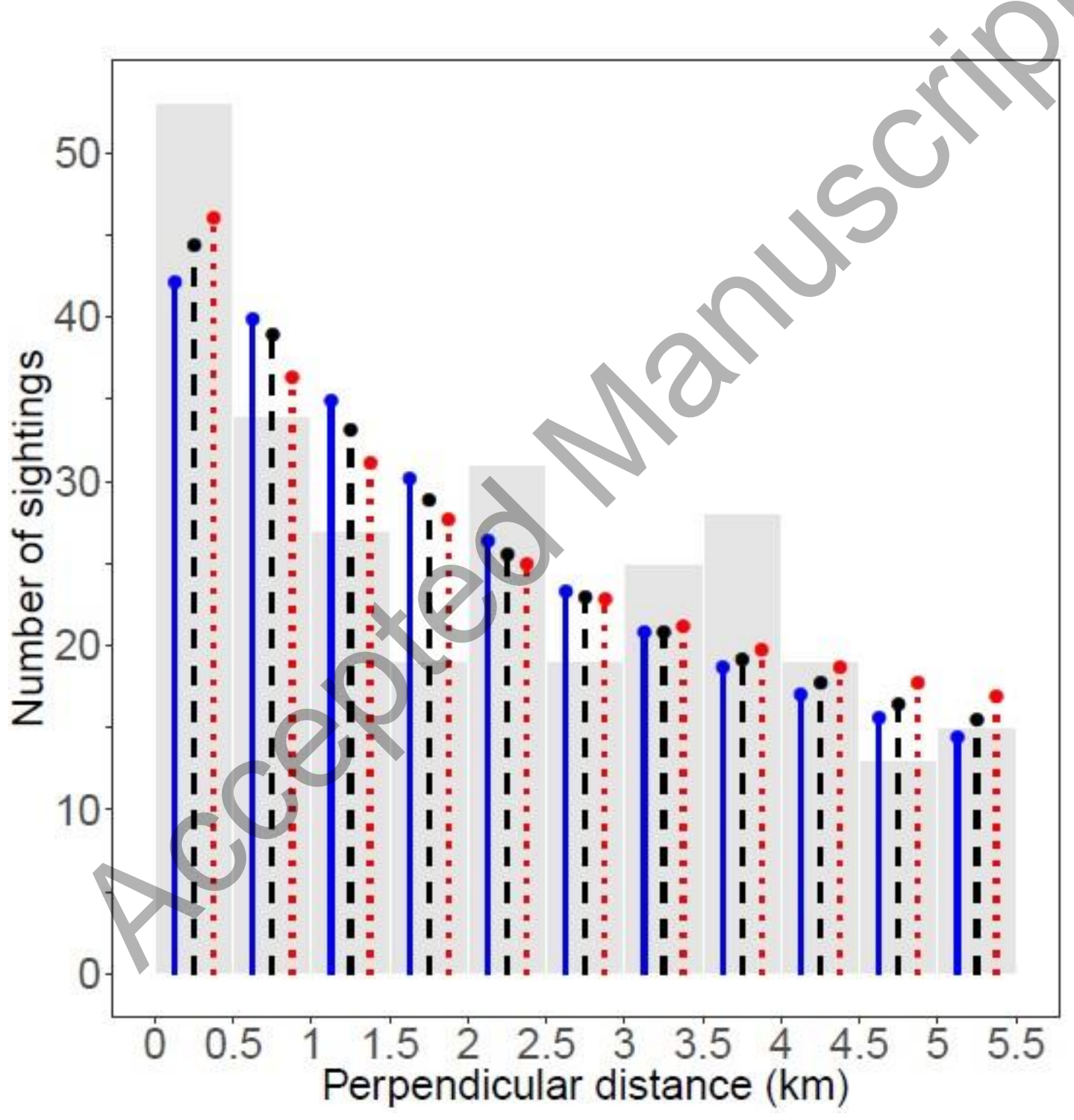


Fig. 4 Estimated detection function for a hypothetically immobile spotted dolphin population for conventional distance sampling, in one (solid line) and two (dashed line) dimensions, and distance sampling with movement (dotted line)

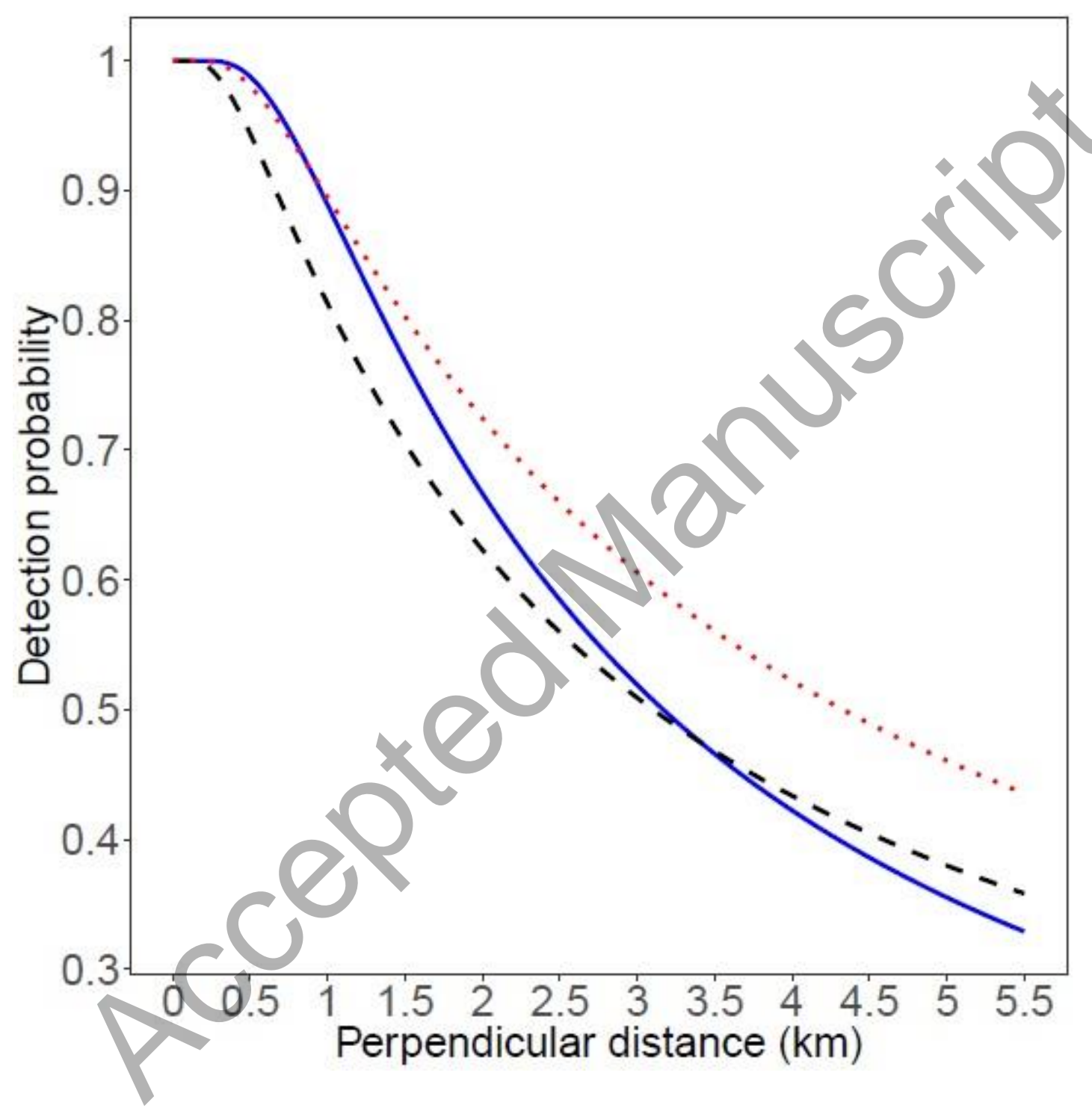


Table 1 Maximum likelihood estimates (Est.) of spotted dolphin school density (per $10^{6} \mathrm{~km}^{2}$ ) with associated percentage coefficient of variation (CV) and $95 \%$ confidence interval $(\mathrm{Cl})$ for conventional distance sampling in one (CDS1D) and two dimensions (CDS2D), and distance sampling with movement in two dimensions (MDS2D)

\begin{tabular}{|r|l|l|l|l|l|l|l|l|l|}
\hline & \multicolumn{3}{|c|}{ CDS1D } & \multicolumn{3}{c|}{ CDS2D } & \multicolumn{3}{c|}{ MDS2D } \\
\hline Yea & Est. & CV & $95 \%$ Cl & Est. & CV & $95 \%$ Cl & Est. & CV & $95 \%$ CI \\
r & & & & & & & & \\
\hline 199 & 107 & 22 & $(700$, & 116 & 18 & $(750$, & 918 & 18 & $(588$, \\
9 & 3 & $\%$ & $1644)$ & 6 & $\%$ & $1581)$ & & $\%$ & $1248)$ \\
\hline 200 & 947 & 23 & $(601$, & 999 & 19 & $(627$, & 787 & 19 & $(492$, \\
0 & & $\%$ & $1493)$ & & $\%$ & $1372)$ & & $\%$ & $1082)$ \\
\hline 200 & 151 & 19 & $(1053$, & 155 & 15 & $(1087$, & 122 & 15 & $(854$, \\
3 & 8 & $\%$ & $2189)$ & 0 & $\%$ & $2013)$ & 3 & $\%$ & $1592)$ \\
\hline 200 & 121 & 24 & $(755$, & 134 & 20 & $(809$, & 105 & 20 & $(636$, \\
6 & 3 & $\%$ & $1947)$ & 2 & $\%$ & $1874)$ & 9 & $\%$ & $1481)$ \\
\hline
\end{tabular}

